\title{
Enhanced Luminescence and Mechanistic Studies on Mn-doped Double Layered Perovskite Phosphors: $\mathrm{Cs}_{4} \mathrm{Mn}_{1-\mathrm{x}} \mathrm{Cd}_{\mathrm{x}} \mathrm{Bi}_{2} \mathrm{Cl}_{12}$
}

\author{
Brenda Vargas, ${ }^{\dagger}$ Diana T. Reyes-Castillo, ${ }^{\dagger}$ Eduardo Coutino-Gonzalez, ${ }^{\perp}$ Citlali Sánchez-Aké, ${ }^{*}$ Carlos

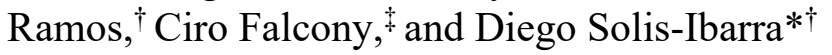 \\ ${ }^{\dagger}$ Instituto de Investigaciones en Materiales, Universidad Nacional Autónoma de México, CU, Coyoacán, 04510, Ciudad de \\ México, México \\ E-mail: diego.solis@unam.com \\ ${ }^{\perp}$ Centro de Investigaciones en Óptica, A. C., Loma del Bosque 115, Colonia Lomas del Campestre, León, Guanajuato, \\ 37150, Mexico \\ * Instituto de Ciencias Aplicadas y Tecnología, Universidad Nacional Autónoma de México, CU, Coyoacán, 04510, Ciudad \\ de México, México \\ ¥ Departamento de Física, Centro de Investigación y de Estudios Avanzados del IPN, Instituto Politécnico Nacional 2508, \\ San Pedro Zacatenco, Gustavo A. Madero Cuidad de México, 07360, México
}

\begin{abstract}
Halide perovskites offer great promise for optoelectronic applications, but stability issues continue to hinder its implementation and long-term stability. The stability of all-inorganic halide perovskites and the inherent quantum confinement of low dimensional perovskites can be harnessed to synthesize materials with high PL efficiency. An example of such materials is the recently reported new family of layered double perovskites, $\mathrm{Cs}_{4} \mathrm{Mn}_{1-\mathrm{x}} \mathrm{Cd}_{\mathrm{x}} \mathrm{Bi}_{2} \mathrm{Cl}_{12}$. Herein, we report a new synthetic procedure that enhances the maximum PLQY of this family materials to up $79.5 \%$, a $20 \%$ enhancement from previous reports and the highest reported for a Mn-doped halide perovskite. Importantly, stability tests demonstrate that these materials are very stable towards humidity, UV irradiation, and temperature. Finally, we investigated the photophysics, the effects of magnetic coupling and temperature in the PL efficiency and proposed a mechanism for the emission process. Our results highlight the potential of this family of materials and related layered all-inorganic perovskites for solid-state lighting and optoelectronic applications.
\end{abstract}

\section{Introduction}

Halide perovskites extraordinary structural and electronic tunability has motivated the quest for applications in optoelectronic devices beyond solar cells, such as light-emitting diodes, ${ }^{1,2}$ X-ray detectors, ${ }^{3,4}$ and catalysts. ${ }^{5,6}$ However, three dimensional (3D) halide perovskites with general formula $\mathrm{ABX}_{3}$ have limited composition diversity. ${ }^{7}$ In contrast, their two-dimensional (2D) counterparts significantly expand the perovskite chemical diversity, allowing further tuning of the optoelectronic properties. ${ }^{8}$ The dimensional reduction of halide perovskites can be achieved by introducing a large organic cation that separates the inorganic layers, resulting in the family of materials commonly known as organic-inorganic hybrid perovskites, or by replacing $\mathrm{B}^{2+}$ with a metal in a different oxidation state, such as $\mathrm{Bi}^{3+}$ or $\mathrm{Sb}^{3+}$. The difference in charge gives rise to vacancies that generate a $2 \mathrm{D}$ network, known as a defective perovskite or $<111>$-oriented layered perovskite $\mathrm{A}_{3} \mathrm{M}_{2} \mathrm{X}_{9}$ where $\mathrm{M}=\mathrm{Bi}$, Sb (Figure 1). On one hand, this heterovalent substitution allows the replacement of lead and expands the possible applications of halide double perovskites. ${ }^{9-12}$ On the other hand, the dimensional reduction of the inorganic sublattice in $2 \mathrm{D}$ perovskites results in electronic confinement within the layers, with the added advantage that, in contrast to perovskite nanocrystals, stabilization is not required, and the resulting materials tend to be more stable. Further, dimensional reduction typically results in structures that have large band gaps and less dispersive bands.
Consequently, these materials tend to be excitonic in nature with high exciton binding energies. ${ }^{13,14}$ Such properties are highly desirable in phosphors for light-emitting diodes applications and have been harnessed for the synthesis of new materials with near-unity photoluminescence quantum yields (PLQYs). ${ }^{12,15-17}$

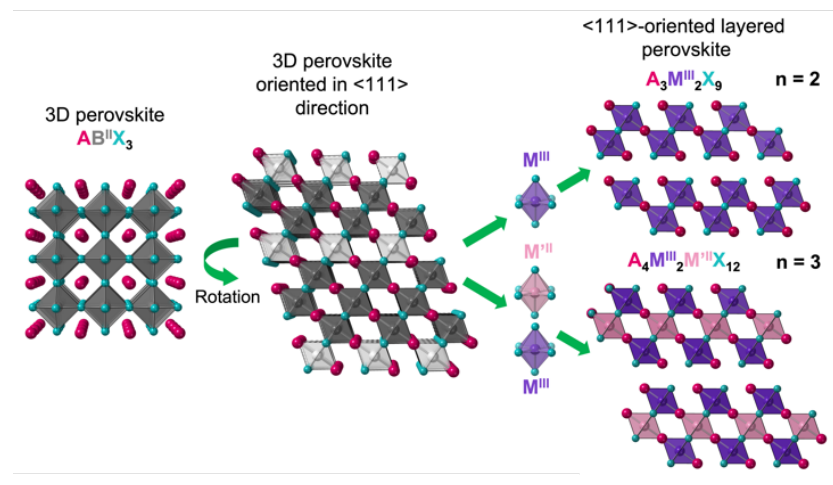

Figure 1. Schematic representation of the relationship between layered $<111>$-oriented perovskites and $3 \mathrm{D}$ perovskites. By replacing $\mathrm{B}^{\mathrm{II}}$ metal with a trivalent metal $\mathrm{M}^{\mathrm{III}}(\mathrm{n}=2)$ or a combination of divalent $\mathrm{M}^{\text {III }}$ and trivalent metals $(\mathrm{n}=3)$ the layered structure can be obtained.

Another alternative to harness the excitonic nature of these materials is the introduction of doping agents or impurities such 
as transition metals or lanthanides. ${ }^{9,18-21}$ Among the transition metals, $\mathrm{Mn}^{2+}$ has been extensively investigated as a dopant in many semiconductor hosts for photoluminescent and electroluminescent applications. ${ }^{22}$ Divalent manganese ions act as recombination centers for electron-hole pairs and exhibit a typical photoluminescence emission centered at $\sim 590 \mathrm{~nm}$. This emission originates from the parity and spin forbidden ${ }^{4} \mathrm{~T}_{1}(\mathrm{G})$ to ${ }^{6} \mathrm{~A}_{1}(\mathrm{~S})$ transition in the $3 \mathrm{~d}$ shell of the octahedrally coordinated $\mathrm{Mn}^{2+}$ ions. ${ }^{22}$ Generally, as the activation of spin relaxation of $\mathrm{Mn}^{2+}$ is induced by the energy transfer from the host exciton to $\mathrm{Mn}^{2+}$, the emission strongly depends on the band-edge position and bandgap of the host. ${ }^{9}$

High photoluminescence efficiencies have been achieved by doping 2D layered perovskites and lower dimension lead halides with $\mathrm{Mn}^{2+} \cdot{ }^{23,24}$ For example, Zhang et al. synthesized 2D single-layered $\mathrm{Mn} @ \mathrm{EA}_{2} \mathrm{PbBr}_{4}(\mathrm{EA}=$ ethylammonium) with a $78 \%$ PLQY. ${ }^{25}$ However, as the organic component in the materials usually decreases their stability, great effort has been devoted to the search of inorganic materials with improved stability towards light, moisture, oxygen, and temperature. $\mathrm{Mn}^{2+}$ doping of $\mathrm{CsPbCl}_{3}$ nanocrystals has also been extensively studied, ${ }^{19,26-30}$ leading to PLQYs of up to $70 \%$. $^{31}$ However, $\mathrm{CsPbCl}_{3}$ nanocrystals are not very stable and tend to degrade rather rapidly, especially when out of the protecting colloidal environment. $^{32-34}$

Recently, P.M. Woodward and collaborators reported the optoelectronic and magnetic properties of a new halide perovskite family $\mathrm{Cs}_{4} \mathrm{Cd}_{1-\mathrm{x}} \mathrm{Mn}_{\mathrm{x}} \mathrm{Bi}_{2} \mathrm{Cl}_{12}$. A remarkable PLQY of $57 \%$ was achieved for a Mn content of $\mathrm{x}=0.27$. The high PLQY was attributed to an efficient energy transfer from $\mathrm{Bi}^{3+}$ sensitizers to $\mathrm{Mn}^{2+}$ activators where incorporation of $\mathrm{Cd}^{2+}$ serves to electronically isolate $\mathrm{Mn}-\mathrm{Bi}-\mathrm{Mn}$ networks, increasing radiative recombination on $\mathrm{Mn}_{2+}$ sites. ${ }^{35}$ The solid solutions $\mathrm{Cs}_{4} \mathrm{Cd}_{1-\mathrm{x}} \mathrm{Mn}_{\mathrm{x}} \mathrm{Bi}_{2} \mathrm{Cl}_{12}$ acquire a greater importance among the emerging family of layered double perovskites as PL emission in the materials reported so far, is either completely quenched or very low in intensity at room temperature. ${ }^{36,37}$ This emphasizes the importance of this family to further understand the optoelectronic properties of layered-double perovskites for future applications in LEDs and phosphors. Further studies on the PL quenching mechanism at room temperature and at low temperatures as well as stability tests are necessary.

Herein, we report the photophysical characterization and a proposed quenching mechanism at room temperature by exchange interaction between $\mathrm{Mn}^{2+}$ of $2 \mathrm{D}$ perovskites with general formula $\mathrm{Cs}_{4} \mathrm{Cd}_{1-\mathrm{x}} \mathrm{MnxBi}_{2} \mathrm{Cl}_{12}$ (where $\mathrm{x}$ ranges from 0 to 1) displaying PL centered at $595 \mathrm{~nm}$ and an improved PLQY of up to $79.5 \%$, a $20 \%$ increase from the same previous reported materials and the highest reported for a Mn-doped perovskite. ${ }^{25,31,38,39}$ The facile synthesis and excellent stability to humidity, air, temperature and UV light irradiation makes these materials, and related all-inorganic 2D perovskites, promising candidates for their use as phosphors in lightemitting devices.

\section{Results and Discussion \\ Synthesis, characterization and stability tests}

We synthesized microcrystalline powders of this family of solid solutions with general formula $\mathrm{Cs}_{4} \mathrm{Cd}_{1-\mathrm{x}} \mathrm{Mn}_{\mathrm{x}} \mathrm{Bi}_{2} \mathrm{Cl}_{12}(\mathrm{x}=0$ -1 ), in large quantities (up to gram scale), by precipitation of the metal halides $\left(\mathrm{CsCl}, \mathrm{MnCl}_{2}, \mathrm{CdCl}_{2}\right.$ and $\left.\mathrm{BiCl}_{3}\right)$ in concentrated hydrochloric acid (see SI for more information). The Cd-only $(\mathrm{x}=0)$ material is a white, microcrystalline powder, and as Mn concentration increases, the material turns to a light-pink color characteristic of $\mathrm{Mn}^{2+}$-containing solids. In agreement to the previous report, powder X-ray diffraction (PXRD) patterns showed that the intermediate materials are pure and preserve the same trigonal $R \overline{3} \mathrm{~m}$ space group and very similar cell constants, with the expected decrease in cell parameters as the Mn-content increases (Figure 2A-B, S1). Inductively Couple Plasma-Optical Emission Spectroscopy (ICP-OES) showed that the composition of the final materials almost perfectly matched that of the desired stoichiometry (Figure 2C) and confirms the full solubility of $\mathrm{Mn}^{2+}$ within the $\mathrm{Cs}_{4} \mathrm{Cd}_{1-\mathrm{x}} \mathrm{Mn}_{\mathrm{x}} \mathrm{Bi}_{2} \mathrm{Cl}_{12}$ family of materials. The latter is notable, as Woodward et. al. previously reported difficulties in controlling the amount Mn with their experimental procedure, further and more generally, a precise control of $\mathrm{Mn}$ incorporation and large doping levels into the crystal lattice are important parameters that are hard to achieve for Mn-doped nanostructures such as $\mathrm{Mn} @ \mathrm{CsPbBr}_{3}$ nanocrystals, and other semiconductor quantum dots, or hybrid perovskites. ${ }^{19,40,41}$
A)

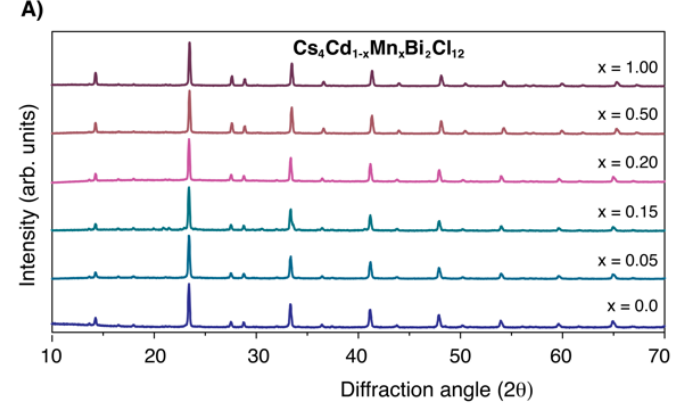

B)

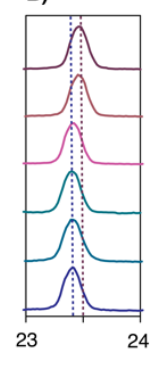

C)

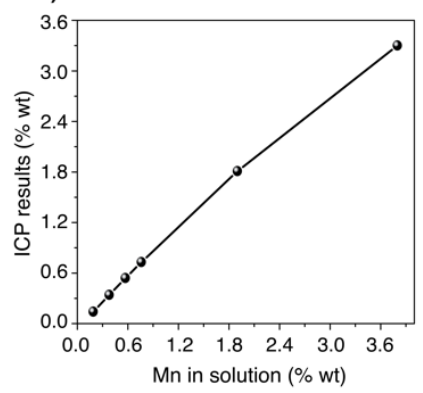

D)

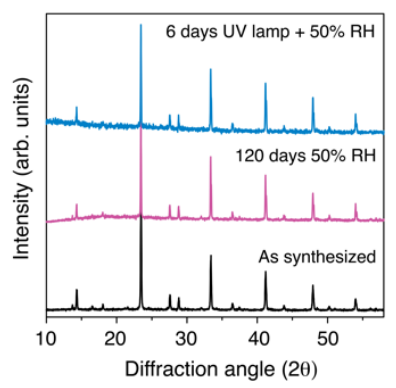

Figure 2. A) PXRD diffractograms of the $\mathrm{Cs}_{4} \mathrm{Cd}_{1-\mathrm{x}} \mathrm{Mn}_{\mathrm{x}} \mathrm{Bi}_{2} \mathrm{Cl}_{12}$ family of $<111>$-oriented layered perovskites with increasing $\mathrm{Mn}$ content (x). B) Displacement in the peak at $\sim 23.5^{\circ}$ corresponding to the (110) diffraction plane as a function of Mn content. C) $\mathrm{Mn}$ content in the materials determined by ICP-OES compared with the amount of Mn added to the solution during the synthesis, and D) PXRD of the sample with $\mathrm{x}=0.1$ after exposure to a relative humidity (RH) of 50\% for 120 days and after a 6 days UV ( 375 $\mathrm{nm})$ irradiation.

After confirmation of the structure, we evaluated the stability of these materials towards humidity, light and temperature. First, thermogravimetric analysis (TGA) and differential scanning calorimetry (DSC) were carried out on the powder samples. The thermal decomposition of the samples started at 
$\sim 300{ }^{\circ} \mathrm{C}$, regardless of Mn content, and the DSC results showed no phase transition in a temperature range of 25 to $330{ }^{\circ} \mathrm{C}$ (Figures S2, S3). Also, the materials exhibited no sign of decomposition after storage in humid air $(\sim 50 \%$ relative humidity) for up to 4 months or after UV irradiation for 6 days

A)

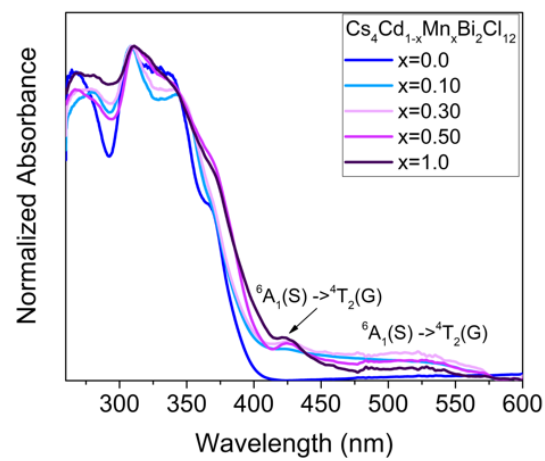

D)

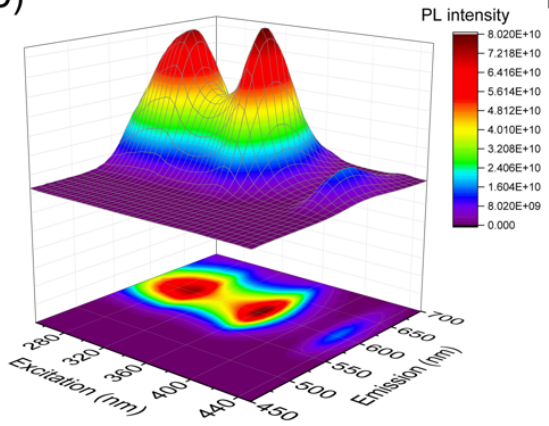

B)

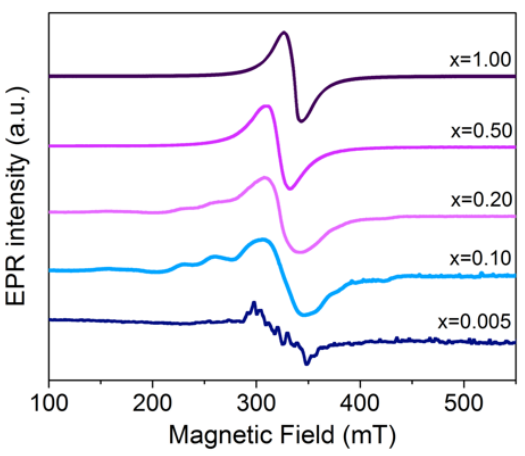

E)

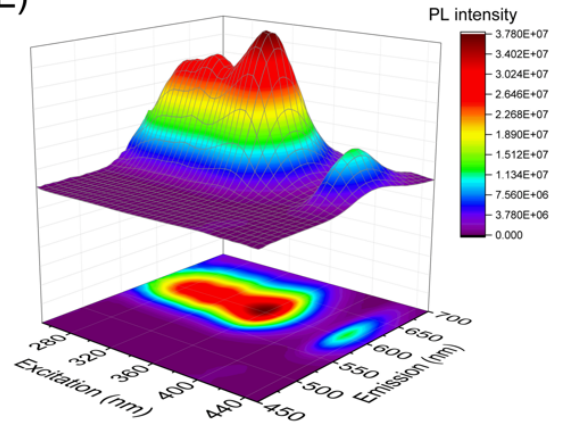

C)

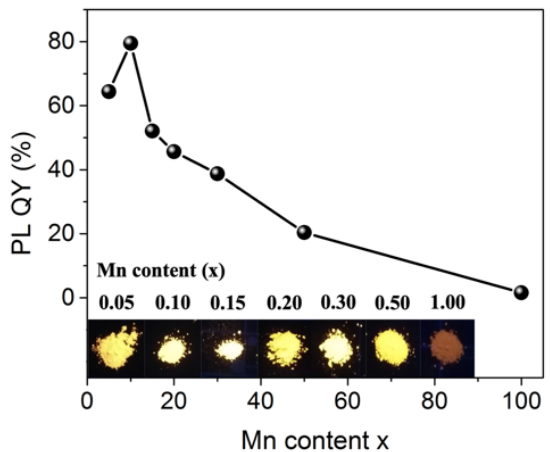

F)

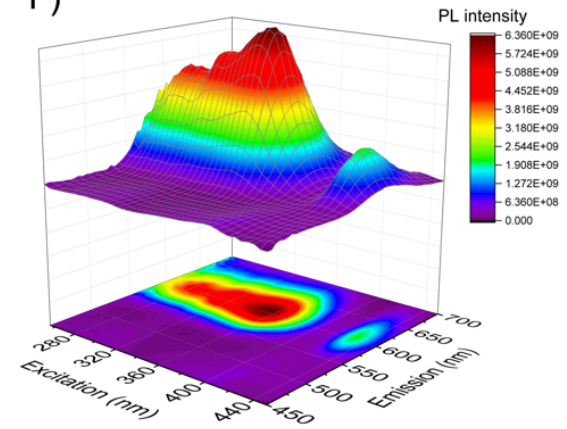

Figure 3. The normalized pseudo-absorbance calculated from the diffuse reflectance measurements (A) and room temperature electron paramagnetic resonance (B) of $\mathrm{Cs}_{4} \mathrm{Cd}_{1-\mathrm{x}} \mathrm{Mn}_{\mathrm{x}} \mathrm{Bi}_{2} \mathrm{Cl}_{12}$ with different $\mathrm{Mn}^{2+}$ concentration $(\mathrm{x}=0.05,0.10,0.20,0.50$ and 1.0). C) PLQY of $\mathrm{Cs}_{4} \mathrm{Cd}_{1-\mathrm{x}} \mathrm{Mn}_{\mathrm{x}} \mathrm{Bi}_{2} \mathrm{Cl}_{12}$ as a function of $\mathrm{Mn}^{2+}$ content, and photoexcitation $3 \mathrm{D}$ mapping of $\mathrm{Cs}_{4} \mathrm{Cd}_{1-\mathrm{x}} \mathrm{Mn}_{\mathrm{x}} \mathrm{Bi}_{2} \mathrm{Cl}_{12}$ with $\mathrm{x}=0.10(\mathrm{D}), \mathrm{x}=0.3(\mathrm{E})$ and $\mathrm{x}=1.0(\mathrm{~F})$.

\section{Absorption and photoluminescence characterization}

To gain insight into the optical properties of these materials, we studied their UV-Vis absorption and PL properties. UV-Vis diffuse reflectance measurements were converted to pseudoabsorbance by the Kubelka-Munk transformation (see SI for complete details). Figure $3 \mathrm{~A}$ of the normalized absorbance of samples with increasing $\mathrm{Mn}^{2+}$ content displays a charge transfer band in the UV region associated with the $6 s^{2} \rightarrow 6 s^{1} p^{1}$ transitions of the $\left[\mathrm{BiCl}_{6}\right]^{3-}$ octahedra. ${ }^{35,39}$ This transition can split into a doublet if a dynamical Jahn Teller distortion is acting on the ion, which explains the two bands at $~ 310$ and $340 \mathrm{~nm}^{35}$ The band edge absorption slightly shifts with Mn incorporation (band gap changes from $3.2 \mathrm{eV}$ for the Mn-only material to 3.1 $\mathrm{eV}$ for the Cd-only material), which suggests that $\mathrm{Mn}$ incorporation has a minimal effect on the band edge (Figure 3A, S4 and Table S3). These results differ with Woodward, as the absorption edge in their materials shifts from $360 \mathrm{~nm}$ (Cd-only material) to $400 \mathrm{~nm}(\mathrm{Mn}$ content $\mathrm{x}=0.55)$, which corresponds to an energy difference of $0.34 \mathrm{eV}$. The broadening of the $6 \mathrm{~s}^{2}$ $\rightarrow 6 \mathrm{~s}^{1} \mathrm{p}^{1}$ transition, also observed in our samples, was ascribed to an increase of the electronic dimensionality with increasing Mn incorporation. ${ }^{42}$ The bands at $430 \mathrm{~nm}$ and $520 \mathrm{~nm}$ correspond to the spin forbidden ${ }^{6} \mathrm{~A}_{1}(\mathrm{~S}) \rightarrow{ }^{4} \mathrm{~T}_{2}(\mathrm{G})$ and ${ }^{6} \mathrm{~A}_{1}(\mathrm{~S})$ $\rightarrow{ }^{4} \mathrm{~T}_{1}(\mathrm{G})$ transitions, respectively. ${ }^{43-45}$ Usually, these transitions are rarely observed as they are forbidden in octahedral coordination, but magnetic coupling between $\mathrm{Mn}^{2+}$ pairs or a symmetry breaking contribution can relax the spin and parity selection rules, respectively. ${ }^{40,46-48}$

The photoexcitation 3D mapping for $\mathrm{x}=0.10,0.30$ and 1.0 noticeably evolve with Mn content (Figure S6) with a new band forming at $320 \mathrm{~nm}$ as Mn concentration increases (Figure 3DF). The intensity of the $430 \mathrm{~nm}$ band corresponding to the ${ }^{6} \mathrm{~A}_{1}(\mathrm{~S}) \rightarrow{ }^{4} \mathrm{~T}_{2}(\mathrm{G})$ transition, steadily increases with increasing Mn content due to the larger amount of $\mathrm{Mn}^{2+}$ coupled pairs. ${ }^{44}$ The emission and absorption spectra of the $\mathrm{Mn}^{2+}$-containing samples are quite similar (Figure S7) suggesting that $\mathrm{Mn}^{2+}$ emission originates from the absorption of the host. ${ }^{49,50}$

The PL emission spectra for $\mathrm{x}=0.1$ (Figure 3D, S5 and S7) shows a peak centered at $595 \mathrm{~nm}$ with an FWHM of approximately $65 \mathrm{~nm}$. The characteristic orange emission is attributed to the spin and parity forbidden ${ }^{4} \mathrm{~T}_{1}(\mathrm{G}) \rightarrow{ }^{6} \mathrm{~A}_{1}(\mathrm{~S})$ transition within the $3 \mathrm{~d}$ shell of the octahedrally coordinated $\mathrm{Mn}^{2+}{ }^{25,40,47}$ The emission peak and FWHM of PL spectra of Mn-containing samples are independent of excitation wavelength (Figure 3D-E-F, S5), which indicates a single radiative decay pathway in these samples. Interestingly, in this family of materials, band edge emission was not observed, unlike in $\mathrm{Mn} @ \mathrm{CsPbX}_{3}$ nanocrystals $(\mathrm{X}=\mathrm{Br}, \mathrm{Cl})$ or $\mathrm{Mn}^{2+}$ doped hybrid lead perovskites, where both band edge and $\mathrm{Mn}^{2+}$ emissions are generally observed. ${ }^{51}$ Band edge emission is absent in double perovskites, a behavior more similar to II-VI chalcogenides than lead perovskites. ${ }^{37,46} \mathrm{R}$. Bakthavatsalam and collaborators probe the effect of confinement on enhancing exciton- $\mathrm{Mn}^{2+}$ exchange coupling interaction in bulk 2D layered 
perovskites. They found that higher confinement in 2D perovskites enhances the dopant-host carrier exchange interactions allowing a much higher dopant energy-transfer rate than the band edge recombination rate..$^{52}$ The layered nature of these materials could have a similar effect in these materials, where the energy transfer from the host to the dopant ion overshadows the band edge exciton recombination, indicating an efficient energy transfer from the host lattice to $\mathrm{Mn}^{2+}$ centers. ${ }^{26,53,54}$ As previously mentioned, the excitonic nature of similar $<111>$-oriented halide perovskites has been studied by several groups. Particularly, B. W. Wessels and collaborators proposed that electron-phonon interactions in some $<111>$ oriented perovskites with $\mathrm{n}=2$, induce small polarons that result in the trapping of excitons by the lattice. Based on these results, it is highly likely that this family of layered materials with $\mathrm{n}=3, \mathrm{Cs}_{4} \mathrm{Cd}_{1-x} \mathrm{Mn}_{x} \mathrm{Bi}_{2} \mathrm{Cl}_{12}$, are excitonic. Further experiments will have to be conducted in order to clarify whether STEs are also formed for these materials. ${ }^{13,14}$

Generally, high $\mathrm{Mn}^{2+}$ doping concentrations redshifts the emission due to magnetic interactions between neighboring $\mathrm{Mn}^{2+}$ ions. ${ }^{55,56}$ However in these materials, the PL emission maximum shifts only $5 \mathrm{~nm}$ in the entire composition range (Figure S8). This can likely be due to the relatively large MnMn distances, which at the shortest distance is $\sim 7.5 \AA$ (Figure S9), probably precluding direct exchange between $\mathrm{Mn}$ centers. ${ }^{57}$ PLQY measurements (Figure 3C) indicate that the maximum efficiency of $79.5 \%$ is achieved for the material with $\mathrm{x}=0.1$. For higher concentrations, the energy transfer between radiative centers leads to non-radiative transitions or concentration quenching. Noteworthy, our champion material shows a 20\% PLQY improvement over the reported materials by Woodward. We attributed the difference to two main factors, first, to the synthetic procedure, as ours uses metal halides instead of oxides, which are more soluble and which also provide a better control over the final Mn-concentration; and second, to the difference in purification procedure, which avoided the use of alcohols and. To the best of our knowledge, $79.5 \%$ is the highest reported PLQY for Mn-doped halide perovskites, including Mn-doped $\mathrm{CsPbBr}_{3}$ and $\mathrm{CsPbCl}_{3}$ nanocrystals, ${ }^{31,41}$ and is also higher than Mn-doped quantum dots $(70 \%)^{48}$ or $\mathrm{Mn}$-doped $\mathrm{Mn}^{2+}$-doped hybrid perovskites $(78 \%)^{25,50,58,59}$

To probe the local environment of paramagnetic ions and get further insights on the coupling of $\mathrm{Mn}^{2+}$ centers in $\mathrm{Cs}_{4} \mathrm{Cd}_{1}$ ${ }_{x} \mathrm{Mn}_{\mathrm{x}} \mathrm{Bi}_{2} \mathrm{Cl}_{12}$, we performed electron paramagnetic resonance (EPR) experiments. The EPR spectra (Figure 3B), can be divided into three groups: for $\mathrm{x}=0.005$, the hyperfine structures, characteristic of isolated $\mathrm{Mn}^{2+}$ ions, are clearly observed. For $0.005<\mathrm{x}<0.2$, the hyperfine lines merge together into a broad signal, indicating that $\mathrm{Mn}^{2+}$ ions are no longer diluted and interact between each other. At these concentrations, the coupling becomes important and the hyperfine lines start to dissipate. For $\mathrm{x}>0.5$, the hyperfine structure completely disappears and the spectra consist of a single broad line with a narrower linewidth, showing that in the latter range the coupling becomes stronger than the hyperfine interaction..$^{60,61}$ These results confirm that the enhanced intensity of the forbidden ${ }^{6} \mathrm{~A}_{1}(\mathrm{~S}) \rightarrow{ }^{4} \mathrm{~T}_{1}(\mathrm{G})$ and ${ }^{6} \mathrm{~A}_{1}(\mathrm{~S}) \rightarrow{ }^{4} \mathrm{~T}_{2}(\mathrm{G})$ transitions can be associated to a $\mathrm{Mn}^{2+}-\mathrm{Mn}^{2+}$ coupling. Interestingly, even in the Mn-diluted regime $(x=0.005)$, the signal is notably broadened (Figure 3B) suggesting that even at low concentrations, some of the $\mathrm{Mn}^{2+}$ ions are interacting with each other. As above-mentioned, an additional coupling interaction different from a direct exchange, such as a longdistance exchange or electric multipolar interaction, is probably taking place. Indeed, an antiferromagnetic interaction between $\mathrm{Mn}^{2+}$ centers was reported by P.M. Woodward. ${ }^{35}$ Y. Liu and collaborators determined that $\mathrm{Mn}-\mathrm{Mn}$ energy transfer is predominantly controlled by dipole-dipole interactions instead of spin-exchange interactions or by the average distance of $\mathrm{Mn}^{2+}$ pairs in other semiconductors. ${ }^{62}$ Further EPR studies can help determine each contribution since EPR spectra can be modelled with a Hamiltonian that takes into account contributions from spin-exchange, multipolar, Zeeman and single ion interactions.

\section{Mechanistic studies of the $\mathrm{Cs}_{4} \mathrm{Cd}_{1-\mathrm{x}} \mathbf{M n x B i}_{2} \mathrm{Cl}_{12}$ family}

To understand the photoluminescence decay mechanism of these perovskites, time-resolved and temperature dependentphotoluminescence experiments were conducted on the samples. Unfortunately, due to low PL intensity, we were not able to carry lifetime or variable temperature measurements in the Cd-only material $(\mathrm{x}=0)$. The materials emission (Figure 4A) displays an exponential decay curve that can be fitted with a bi-exponential function for samples with $\mathrm{x}<0.2$ and a triexponential function for $\mathrm{x}>0.5$ (see Supporting Information). First, for samples with $\mathrm{x}<0.2$, the total decay pathway has a fast and a slow component with a decay time $\tau_{1}$ and $\tau_{2}$, respectively (for the $\mathrm{x}=0.05$ material, $\tau_{1}=45.8 \mu \mathrm{s}$ and $\tau_{2}=396.6$ $\mu \mathrm{s})$. The difference in magnitude between decay times is of one order of magnitude, both values in the order of microseconds in agreement with the forbidden nature of the transition. ${ }^{22,40}$ Consistent with the EPR results, the slow component $\tau_{2}$ can be assigned to the emission of the isolated $\mathrm{Mn}^{2+}$ ions emission and the fast component $\tau 1$ to the coupled $\mathrm{Mn}^{2+}$ centers as the spin selection rule is relaxed for these magnetically interacting $\mathrm{Mn}^{2+}$ pairs (table S4) ${ }^{48,55}$ The contribution to the total decay intensity from each decay pathway is reflected on the amplitude coefficient $A_{x}$. As shown in table S4, the contribution from $\mathrm{Mn}^{2+}$ pairs increases as the Mn concentration increases and simultaneously, the contribution from isolated $\mathrm{Mn}^{2+}$ decreases. The decay time of both processes decreases with increasing $\mathrm{Mn}$ content (Figure S12) and when $\mathrm{x}=0.5$, a third decay pathway comes into play with a decay lifetime $\tau_{3}=1.2 \mu \mathrm{s}$. Both observations are likely explained by the introduction of new non-radiative pathways that are also responsible for lower PLQYs at high Mn concentrations (concentration quenching by $\mathrm{Mn}^{2+}$ coupling). ${ }^{46,63}$

Temperature-dependent PL can provide useful information on crystal field strength of the dopant, energy transfer between excitons and dopants and phonon coupling. ${ }^{40}$ For $\mathrm{Cs}_{4} \mathrm{Cd}_{1}$. ${ }_{x} \mathrm{Mn}_{\mathrm{x}} \mathrm{Bi}_{2} \mathrm{Cl}_{12}$, the ${ }^{4} \mathrm{~T}_{1}(\mathrm{G}) \rightarrow{ }^{6} \mathrm{~A}_{1}(\mathrm{~S})$ transition for both materials $(\mathrm{x}=0.05$ and 0.2$)$ underwent a continuous redshift and emission narrowing (Figure 4B-C) when the temperature decreased. The crystal field strength is enhanced as a consequence of thermal lattice contraction, which results in the shrinkage of the ${ }^{4} \mathrm{~T}_{1}(\mathrm{G})$ $\rightarrow{ }^{4} \mathrm{~A}_{1}(\mathrm{~S})$ transition and the energy peak redshift. An additional factor must be affecting the host lattice crystal splitting since at $12 \mathrm{~K}$, the sample with $\mathrm{x}=0.20$ has a lower energy peak than the $\mathrm{x}=0.05$ sample, indicating that the crystal field of the latter is larger, but at $300 \mathrm{~K}$ both samples reach the same energy. The larger concentration of $\mathrm{Mn}$ ions in the sample with $\mathrm{x}=0.20$ might cause a greater lattice strain than in the $\mathrm{x}=0.05$ sample, and could probably originate a larger crystal splitting. ${ }^{64}$ The linear change in FWHM should be mostly due to phonon broadening (Figure 4-F). Both samples $(x=0.20$ and 0.05$)$ 
exhibit two quenching processes (Figure 4B-D), one for temperatures below $100 \mathrm{~K}$, and the second for higher temperatures. The temperature dependence of the luminescence intensity can be described by the Arrhenius equation $\mathrm{I}_{\mathrm{Mn}}(\mathrm{T})=$ $\mathrm{I}_{0} /(1+\mathrm{A} \exp (-\mathrm{Ea} / \mathrm{kT}))$ where $\mathrm{I}_{0}$ is PL intensity at $0 \mathrm{~K}, \mathrm{I}(\mathrm{T})$ is the $\mathrm{PL}$ intensity at different temperatures, $\mathrm{Ea}$ is the activation energy of thermal quenching, $\mathrm{A}$ is a constant related to the ratio of the nonradiative rate to the radiative rate and $\mathrm{kB}$ is the
Boltzmann constant (more information in SI). ${ }^{65,66}$ The low temperature quenching, yields an activation energy of $5 \mathrm{meV}$ and $10 \mathrm{meV}$ for $\mathrm{x}=0.05$ and $\mathrm{x}=0.20$, respectively. This process occurs even at ambient temperature, reducing the energy transfer efficiency to the $\mathrm{Mn}^{2+}$ and therefore the luminescence
A)

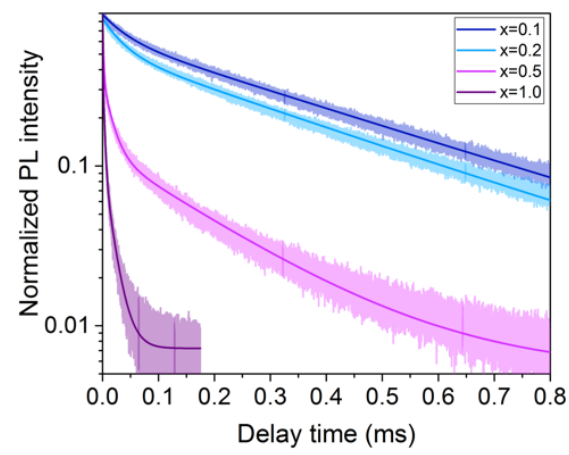

D)

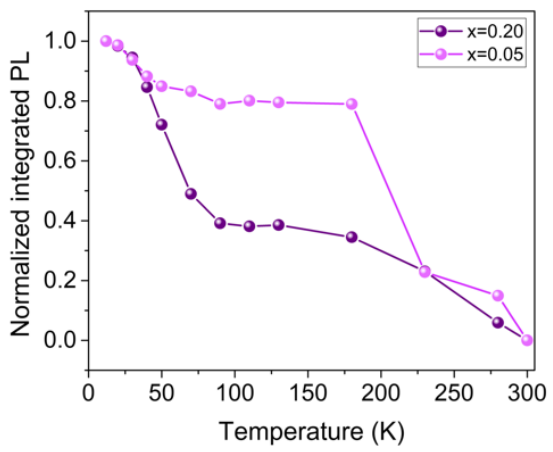

B)

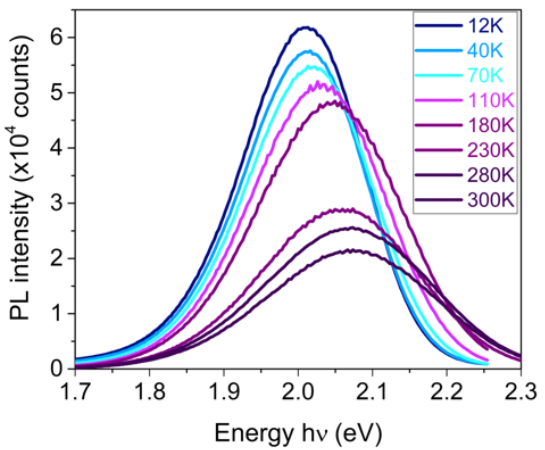

E)

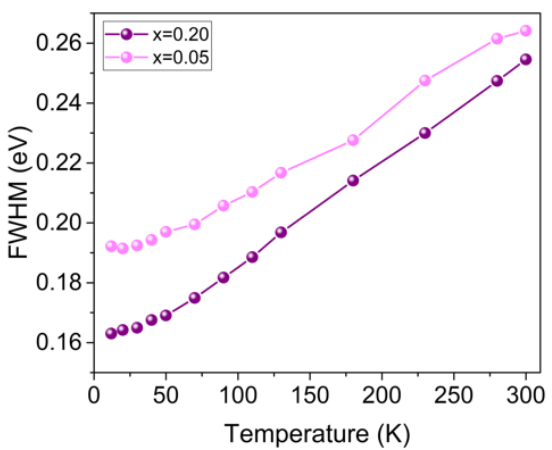

C)

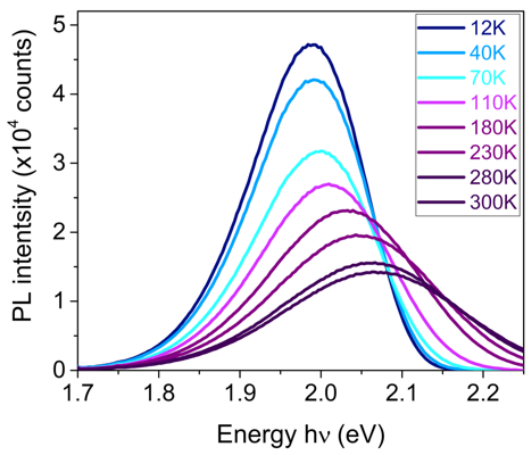

F)

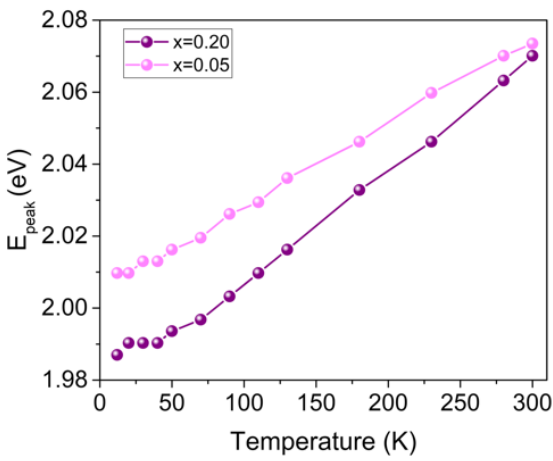

Figure 4. A) Room temperature, time-resolved PL spectra and the corresponding fitting for $\mathrm{x}=0.1,0.2,0.5$ and 1.0 . The excitation wavelength was $325 \mathrm{~nm}$ and the emission recorded at $605 \mathrm{~nm}$. (B) temperature-dependent PL spectra for $\mathrm{x}=0.05$, and $(\mathrm{C})$ temperaturedependent PL for $\mathrm{x}=0.20$. D) normalized integrated PL for $\mathrm{x}=0.05$ and $0.20, \mathrm{E}$ ) and F) FWHM and peak PL for por $\mathrm{x}=0.05$ and 0.2 , respectively.

intensity, $\mathrm{I}(300 \mathrm{~K}) / \mathrm{I}(12 \mathrm{~K})$, in $56 \%$ and $55 \%$ for $\mathrm{x}=0.05$ and $\mathrm{x}$ $=0.20$, respectively. Nonetheless, the intensity quenching percentage is similar to $\mathrm{Mn}^{2+}$ doped $\mathrm{ZnS}$ and $\mathrm{CdS}$ NCs, where excitons are hardly dissociated because of the spatial confinement. ${ }^{67}$ Even if a non-radiative recombination process is activated at room temperature, the quenching values are similar to $\mathrm{Mn}^{2+}$ doped $\mathrm{ZnS}$ and $\mathrm{CdS}$ nanocrystals. A second quenching mechanism is present at higher temperatures but a comparison between samples could not be made

Unlike other halide perovskites, in these materials, we were not able to observe direct band edge radiative decay in the entire range of temperatures and compositions studied $(12-300 \mathrm{~K}$ and $\mathrm{x}=0-1)$. This suggests a very efficient and fast energy transfer from the conduction band to the $\mathrm{Mn}^{2+}$ centers, a behavior closest to doped II-VI NCs than halide perovskites. ${ }^{46}$ To confirm this, a small difference between the host band-edge and $\mathrm{Mn}^{2+}$ based transitions would be expected., ${ }^{968-71}$ To investigate this, we determined the approximate band alignment (see Supporting Information for details), and it was found that the valence and conduction band edges are located at $-5.6 \mathrm{eV}$ and $-2.5 \mathrm{eV}$ respectively. This energy band alignment is very similar to that of $\mathrm{Mn} @ \mathrm{CsPCl}_{3}$ perovskites, which is consistent with an efficient energy transfer. ${ }^{68}$

Based on the results mentioned above and taking into consideration the expected energy diagram for an octahedrally coordinated $\mathrm{Mn}^{2+}$ ion, ${ }^{43}$ we propose a PL mechanism for the $\mathrm{Cs}_{4} \mathrm{Cd}_{1-\mathrm{x}} \mathrm{Mn}_{\mathrm{x}} \mathrm{Bi}_{2} \mathrm{Cl}_{12}$ family of materials (Figure 5). First, upon irradiation, excitons are generated in the host material. Then, several factors contribute to an efficient energy transfer from the host to the $\mathrm{Mn}^{2+}$ centers and a low rate of exciton dissociation: their respective relative energy levels and the quantum confinement derived from the perovskites low dimensional nature (as proposed by Woodward). Since the emission of the material comes from the $\mathrm{Mn}^{2+}$ centers, as the concentration increases, the PLQY also increases due to a growing probability of energy transfer from the host to the $\mathrm{Mn}^{2+}$ centers, reaching a maximum photoluminescence efficiency of $79.5 \%$ for a $\mathrm{Mn}^{2+}$ content of $\mathrm{x}=0.1 .46$ More specifically, the efficient energy transfer to the ${ }^{4} \mathrm{~T} 1(\mathrm{G})$ state of the $\mathrm{Mn}^{2+}$ from which radiative decay to the ${ }^{6} \mathrm{~A} 1(\mathrm{~S})$ state occurs, gives rise to the characteristic orange-red emission. Magnetically coupled $\mathrm{Mn}^{2+}$ pairs in the materials allow for the relaxation of the 
selection rules and a faster and more efficient radiative recombination. As the $\mathrm{Mn}^{2+}$ concentration in the samples further increases, the number of interacting pairs increases, leading to concentration quenching and explaining the decrease in PLQY at higher $\mathrm{Mn}^{2+}$ concentrations. ${ }^{44}$

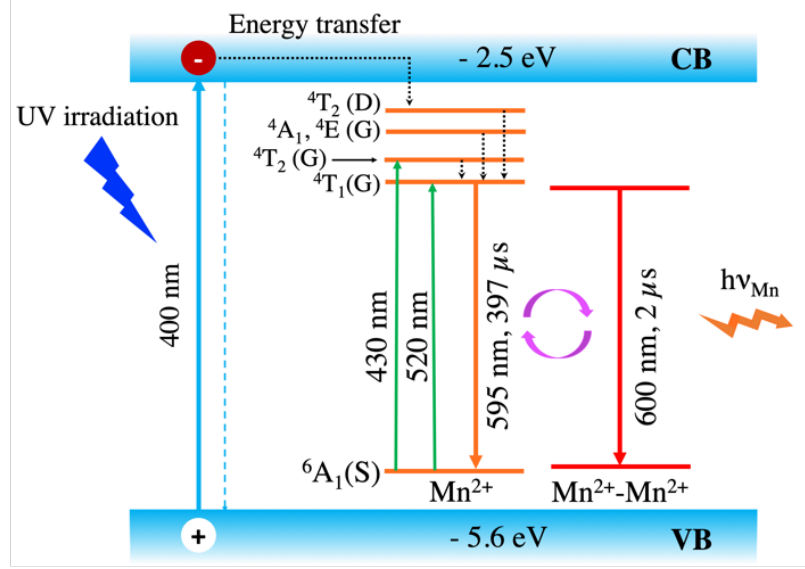

Figure 5. Schematic representation of the PL mechanism of the $\mathrm{Cs}_{4} \mathrm{Cd}_{1-\mathrm{x}} \mathrm{Mn}_{\mathrm{x}} \mathrm{Bi}_{2} \mathrm{Cl}_{12}$ double perovskites. The solid arrows represent absorption and radiative transitions. The dotted arrows are nonradiative transitions. The magenta circled arrows represent exchange interactions between $\mathrm{Mn}^{2+}$ pairs.

\section{Conclusion}

In summary, we demonstrate an improved method to synthesize the recently discovered family of $<111>$-oriented double perovskites: $\mathrm{Cs}_{4} \mathrm{Cd}_{1-\mathrm{x}} \mathrm{Mn}_{\mathrm{x}} \mathrm{Bi}_{2} \mathrm{Cl}_{12}$. The improved method results in an enhanced record PLQY of $79.5 \%$. Further, we studied the photoluminescence properties and quenching mechanisms of this family of materials. Our experiment demonstrates the excitonic properties and remarkable chemical stability of $<111>$-oriented double perovskites, which confer them great potential for optoelectronic applications. The PL efficiency disparity in a previous report of these materials is probably due to a different synthetic procedure, however, more studies should be carried to clarify this. Moreover, our results show that all-inorganic two-dimensional double perovskites are an attractive alternative to 3D perovskites, perovskite nanocrystals, and hybrid organic-inorganic perovskites. Future work will focus on detailing the role of coupling in the emission mechanism and exploring other possible ways to harness the excitonic properties of these materials.

\section{ASSOCIATED CONTENT}

\section{Supporting Information}

The Supporting Information is available free of charge on the ACS Publications website.

Detailed experimental procedures (PDF)

\section{AUTHOR INFORMATION}

\section{Corresponding Author}

*E-mail: $\underline{\text { diego.solis@unam.mx. }}$
Diego Solis-Ibarra: 0000-0002-2486-0967

Funding Sources

The authors thank funding from PAPIIT IA202418 and CONACYT's CB-A1-S-8729.

\section{ACKNOWLEDGEMENTS}

We thank Adriana Tejeda, Eriseth Morales, Lázaro Huerta, Alberto López, Alejandro Pompa, Cain González, Virginia Gómez and Miguel Angel Canseco for technical assistance. We also acknowledge M. Sc. Javier Tadeo Leon from the spectroscopy laboratory LANGEM-Instituto de Geología UNAM for the ICP analysis. B.V. acknowledges the support from CONACYT scholarship (Grant No. 270518).

\section{REFERENCES}

ORCID
Chen, P.; Bai, Y.; Lyu, M.; Yun, J.-H.; Hao, M.; Wang, L. Progress and Perspective in Low-Dimensional Metal Halide Perovskites for Optoelectronic Applications. Sol. RRL 2018, 2, 1700186 (1-28).

Song, Z.; Zhao, J.; Liu, Q. Luminescent Perovskites: Recent Advances in Theory and Experiments. Inorg. Chem. Front. 2019, 6, 2969-3011.

Sytnyk, M.; Deumel, S.; Tedde, S. F.; Matt, G. J.; Heiss, W. A Perspective on the Bright Future of Metal Halide Perovskites for X-Ray Detection. Appl. Phys. Lett. 2019, 115, 190501.

Bin-Bin Zhang, Xin Liu, Bao Xiao, Ahmed Ben Hafsia, Kaige Gao, Yadong Xu, Jian Zhou, and Y. C. Performance X-Ray Detection Based on OneDimensional Inorganic Halide Perovskite $\mathrm{CsPbI}_{3}$. J. Phys. Chem. Lett. 2019, 2, 432-437.

Guo, S.; Zhou, J.; Zhao, X.; Sun, C.; You, S.; Wang, X.; $\mathrm{Su}, \mathrm{Z}$. Enhanced $\mathrm{CO}_{2}$ Photoreduction via Tuning Halides in Perovskites. J. Catal. 2019, 369, 201-208.

Zhu, X.; Lin, Y.; San Martin, J.; Sun, Y.; Zhu, D.; Yan, Y. Lead Halide Perovskites for Photocatalytic Organic Synthesis. Nat. Commun. 2019, 10, 2843 (1-10).

Ortiz-Cervantes, C.; Carmona-Monroy, P.; Solis-Ibarra, D. Two-Dimensional Halide Perovskites in Solar Cells: 2D or Not 2D? ChemSusChem 2019, 12, 1560-1575.

Mao, L.; Stoumpos, K. C.; Kanatzidis, M. G. TwoDimensional Hybrid Halide Perovskites: Principles and Promises. J. Am. Chem. Soc. 2018, 141, 1171-1190.

Luo, B.; Li, F.; Xu, K.; Guo, Y.; Liu, Y.; Xia, Z.; Zhang, J. Z. B-Site Doped Lead Halide Perovskites: Synthesis, Band Engineering, Photophysics, and Light Emission Applications. J. Mater. Chem. C 2019, 7, 2781-2808.

Adhikari, G. C.; Thapa, S.; Zhu, H.; Zhu, P. Mg²+Alloyed All-Inorganic Halide Perovskites for White LightEmitting Diodes by 3D-Printing Method.Adv. Opt. Mater. 2019, 7 , 1900916 (1-9).

Thapa, S.; Adhikari, G. C.; Zhu, H.; Grigoriev, A.; Zhu, P. Zn-Alloyed All-Inorganic Halide Perovskite-Based White Light-Emitting Diodes with Superior Color Quality. Sci.Rep. 2019, 9, 18636 (1-10).

Luo, J.; Hu, M.; Niu, G.; Tang, J. Lead-Free Halide Perovskites and Perovskite Variants as Phosphors toward Light-Emitting Applications. ACS Appl. Mater. Interfaces 2019, 11, 31575-31584.

McCall, K. M.; Stoumpos, C. C.; Kostina, S. S.; Kanatzidis, M. G.; Wessels, B. W. Strong ElectronPhonon Coupling and Self-Trapped Excitons in the Defect Halide Perovskites $\mathrm{A}_{3} \mathrm{M}_{2} \mathrm{I}_{9}(\mathrm{~A}=\mathrm{Cs}, \mathrm{Rb} ; \mathrm{M}=\mathrm{Bi}, \mathrm{Sb})$. Chem. Mater. 2017, 29, 4129-4145.

Correa-Baena, J. P.; Nienhaus, L.; Kurchin, R. C.; Shin, S. S.; Wieghold, S.; Putri Hartono, N. T.; Layurova, M.; Klein, N. D.; Poindexter, J. R.; Polizzotti, A.; Sun, S.; 
Bawendi, M. G.; Buonassisi, T. A-Site Cation in Inorganic $\mathrm{A}_{3} \mathrm{Sb}_{2} \mathrm{I}_{9}$ Perovskite Influences Structural Dimensionality, Exciton Binding Energy, and Solar Cell Performance. Chem. Mater. 2018, 30, 3734-3742.

(15) Connor, B. A.; Leppert, L.; Smith, M. D.; Neaton, J. B.; Karunadasa, H. I. Layered Halide Double Perovskites: Dimensional Reduction of $\mathrm{Cs}_{2} \mathrm{AgBiBr}_{6}$.J.Am. Chem. Soc. 2018, 140, 5235-5240.

(16) Cortecchia, D.; Yin, J.; Petrozza, A.; Soci, C. White Light Emission in Low-Dimensional Perovskites. J. Mat. Chem. C 2019, 7, 4956-4969.

(17) Wang, A.; Guo, Y.; Zhou, Z.; Niu, X.; Wang, Y.; Muhammad, F.; Li, H.; Zhang, T.; Wang, J.; Nie, S.; Deng, Z. Aqueous Acid-Based Synthesis of Lead-Free Tin Halide Perovskites with near-Unity Photoluminescence Quantum Efficiency. Chem. Sci. 2019, 10, 4573-4579.

(18) Ma, J.-P.; Chen, Y.-M.; Zhang, L.-M.; Guo, S.-Q.; Liu, J.D.; Li, H.; Ye, B.-J.; Li, Z.-Y.; Zhou, Y.; Zhang, B.-B.; Bakr, O. M.; Zhang, J.-Y.; Sun, H.-T. Insights into the Local Structure of Dopants, Doping Efficiency, and Luminescence Properties of Lanthanide-Doped $\mathrm{CsPbCl}_{3}$ Perovskite Nanocrystals. J. Mater. Chem. C 2019, 7, 3037-3048.

(19) Das Adhikari, S.; Guria, A. K.; Pradhan, N. Insights of Doping and the Photoluminescence Properties of MnDoped Perovskite Nanocrystals. J. Phys. Chem. Lett. 2019, 10, 2250-2257.

(20) Chen, N.; Cai, T.; Li, W.; Hills-Kimball, K.; Yang, H.; Que, M.; Nagaoka, Y.; Liu, Z.; Yang, D.; Dong, A.; Xu, C.-Y.; Zia, R.; Chen, O. Yb- and Mn-Doped Lead-Free Double-Perovskite $\mathrm{Cs}_{2} \mathrm{AgBiX}_{6} \quad\left(\mathrm{X}=\mathrm{Cl}^{-}, \mathrm{Br}^{-}\right)$ Nanocrystals. ACS Appl. Mater. Interfaces 2019, 11, 16855-16863.

(21) Zhou, Y.; Chen, J.; Bakr, O. M.; Sun, H. T. Metal-Doped Lead Halide Perovskites: Synthesis, Properties, and Optoelectronic Applications. Chem. Mater. 2018, 30, 6589-6613.

(22) Bradshaw, L. R.; Hauser, A.; McLaurin, E. J.; Gamelin, D. R. Luminescence Saturation via $\mathrm{Mn}^{2+}$-Exciton Cross Relaxation in Colloidal Doped Semiconductor Nanocrystals. J. Phys. Chem. C 2012, 116, 9300-9310.

(23) Yangui, A.; Roccanova, R.; Wu, Y.; Du, M. H.; Saparov, B. Highly Efficient Broad-Band Luminescence Involving Organic and Inorganic Molecules in a Zero-Dimensional Hybrid Lead Chloride. J. Phys. Chem. C 2019, 123, 22470-22477.

(24) Yuan, H.; Massuyeau, F.; Gautier, N.; Kama, A. B.; Faulques, E.; Chen, F.; Shen, Q.; Zhang, L.; Paris, M.; Gautier, R. Doped Lead Halide White Phosphors for Very High Efficiency and Ultra-High Color Rendering. Angew. Chemie - Int. Ed. 2020, 59, 2802-2807.

(25) Luo, B.; Guo, Y.; Li, X.; Xiao, Y.; Huang, X.; Zhang, J. Z. Efficient Trap-Mediated $\mathrm{Mn}^{2+}$ Dopant Emission in Two Dimensional Single-Layered Perovskite $\left(\mathrm{CH}_{3} \mathrm{CH}_{2} \mathrm{NH}_{3}\right)_{2} \mathrm{PbBr}_{4}$. J. Phys. Chem. C 2019, 123, 14239-14245.

(26) Guria, A. K.; Dutta, S. K.; Adhikari, S. Das; Pradhan, N. Doping $\mathrm{Mn}^{2+}$ in Lead Halide Perovskite Nanocrystals: Successes and Challenges. ACS Energy Lett. 2017, 2, 1014-1021.

(27) Zhou, C.; Tian, Y.; Khabou, O.; Worku, M.; Zhou, Y.; Hurley, J.; Lin, H.; Ma, B. Manganese-Doped OneDimensional Organic Lead Bromide Perovskites with Bright White Emissions. ACS Appl. Mater. Interfaces 2017, 9, 40446-40451.

(28) Parobek, D.; Roman, B. J.; Dong, Y.; Jin, H.; Lee, E.; Sheldon, M.; Son, D. H. Exciton-to-Dopant Energy Transfer in Mn-Doped Cesium Lead Halide Perovskite Nanocrystals. Nano Lett. 2016, 16, 7376-7380.
(36) Mao, L.; Teicher, S. M. L.; Stoumpos, C. C.; Kennard, R. M.; Decrescent, R. A.; Wu, G.; Schuller, J. A.; Chabinyc, M. L.; Cheetham, A. K.; Seshadri, R. Chemical and Structural Diversity of Hybrid Layered Double Perovskite Halides. J. Am. Chem. Soc. 2019, 141, 19099-19109.

(37) Connor, B. A.; Biega, R.-I.; Leppert, L.; Karunadasa, H. I. Dimensional Reduction of the Small-Bandgap Double Perovskite $\mathrm{Cs}_{2} \mathrm{AgTlBr}_{6}$. Chem. Sci. 2020, Advance Article.

(38) Nila Nandha, K.; Nag, A. Synthesis and Luminescence of Mn-Doped $\mathrm{Cs}_{2} \mathrm{AgInCl}_{6}$ Double Perovskites. Chem. Commun. 2018, 54, 5205-5208.

(39) Majher, J. D.; Gray, M. B.; Strom, T. A.; Woodward, P. M. $\mathrm{Cs}_{2} \mathrm{NaBiCl}_{6}: \mathrm{Mn}^{2+}-\mathrm{A}$ New Orange-Red Halide Double Perovskite Phosphor. Chem. Mater. 2019, 31, 1738-1744.

(40) Lin, J.; Zhang, Q.; Wang, L.; Liu, X.; Yan, W.; Wu, T.; $\mathrm{Bu}$, X.; Feng, P. Atomically Precise Doping of Monomanganese Ion into Coreless Supertetrahedral Chalcogenide Nanocluster Inducing Unusual Red Shift in $\mathrm{Mn}^{2+}$ Emission. J. Am. Chem. Soc. 2014, 136, 4769-4779.

(41) Chen, D.; Fang, G.; Chen, X. Silica-Coated Mn-Doped $\mathrm{CsPb}(\mathrm{Cl} / \mathrm{Br})_{3}$ Inorganic Perovskite Quantum Dots: Exciton-to-Mn Energy Transfer and Blue-Excitable SolidState Lighting. ACS Appl. Mater. Interfaces 2017, 9, 40477-40487.

(42) Vargas, B.; Torres-Cadena, R.; Reyes-Castillo, D. T.; Rodríguez-Hernández, J.; Gembicky, M.; MenéndezProupin, E.; Solis-Ibarra, D. Chemical Diversity in LeadFree, Layered Double Perovskites: A Combined Experimental and Computational Approach. Chem. Mater. 2020, 32, 424-429.

(43) Silva, A. S.; Lourenço, S. A.; Dantas, N. O. Mn Concentration-Dependent Tuning of $\mathrm{Mn}^{2+} \mathrm{d}$ Emission of $\mathrm{Zn}_{1-\mathrm{x}} \mathrm{Mn}_{\mathrm{x}}$ Te Nanocrystals Grown in a Glass System. Phys. Chem. Chem. Phys. 2016, 18, 6069-6076.

(44) Zhang, J.-C.; Zhao, L.-Z.; Long, Y.-Z.; Zhang, H.-D.; Sun, B.; Han, W.-P.; Yan, X.; Wang, X. Color Manipulation of 
Intense Multiluminescence from CaZnOS: $\mathrm{Mn}^{2+}$ by $\mathrm{Mn}^{2+}$ Concentration Effect. Chem. Mater. 2015, 27, 7481-7489. Cheng, X.; Jing, L.; Yuan, Y.; Du, S.; Yao, Q.; Zhang, J.; Ding, J.; Zhou, T. Centimeter-Size Square 2D Layered PbFree Hybrid Perovskite Single Crystal $\left(\mathrm{CH}_{3} \mathrm{NH}_{3}\right)_{2} \mathrm{MnCl}_{4}$ for Red Photoluminescence. CrystEngComm. 2019, 21 , 4085-4091.

(46) Yuan, X.; Ji, S.; De Siena, M. C.; Fei, L.; Zhao, Z.; Wang, Y.; Li, H.; Zhao, J.; Gamelin, D. R. Photoluminescence Temperature Dependence, Dynamics, and Quantum Efficiencies in $\mathrm{Mn}^{2+}$-Doped $\mathrm{CsPbCl}_{3}$ Perovskite Nanocrystals with Varied Dopant Concentration. Chem. Mater. 2017, 29, 8003-8011.

(47) Yang, X.; Pu, C.; Qin, H.; Liu, S.; Xu, Z.; Peng, X. Temperature- and $\mathrm{Mn}^{2+}$ Concentration-Dependent Emission Properties of $\mathrm{Mn}^{2+}$-Doped $\mathrm{ZnSe}$ Nanocrystals. $J$. Am. Chem. Soc. 2019, 141, 2288-2298.

(48) Cao, S.; Zheng, J.; Zhao, J.; Wang, L.; Gao, F.; Wei, G.; Zeng, R.; Tian, L.; Yang, W. Highly Efficient and WellResolved $\mathrm{Mn}^{2+}$ Ion Emission in MnS/ZnS/CdS Quantum Dots. J. Mater. Chem. C 2013, 1, 2540-2547.

(49) Luo, B.; Guo, Y.; Li, X.; Xiao, Y.; Huang, X.; Zhang, J. Z. Efficient Trap-Mediated $\mathrm{Mn}^{2+}$ Dopant Emission in Two Dimensional Single-Layered Perovskite $\left(\mathrm{CH}_{3} \mathrm{CH}_{2} \mathrm{NH}_{3}\right)_{2} \mathrm{PbBr}_{4}$. J. Phys. Chem. C 2019, 123, 14239-14245.

(50) Cortecchia, D.; Mróz, W.; Neutzner, S.; Borzda, T.; Folpini, G.; Brescia, R.; Petrozza, A. Defect Engineering in 2D Perovskite by $\mathrm{Mn}(\mathrm{II})$ Doping for Light-Emitting Applications. Chem 2019, 5, 2146-2158.

(51) Das Adhikari, S.; Guria, A. K.; Pradhan, N. Insights of Doping and the Photoluminescence Properties of $\mathrm{Mn}$ Doped Perovskite Nanocrystals. J. Phys. Chem. Lett. 2019, 10, 2250-2257.

(52) Bakthavatsalam, R.; Biswas, A.; Chakali, M.; Bangal, P. R.; Kore, B. P.; Kundu, J. Temperature-Dependent Photoluminescence and Energy-Transfer Dynamics in $\mathrm{Mn}^{2+}$-Doped $\left(\mathrm{C}_{4} \mathrm{H}_{9} \mathrm{NH}_{3}\right)_{2} \mathrm{PbBr}_{4}$ Two-Dimensional (2D) Layered Perovskite. J. Phys. Chem. C 2019, 123, 47394748.

(53) Pinchetti, V.; Anand, A.; Akkerman, Q. A.; Sciacca, D.; Lorenzon, M.; Meinardi, F.; Fanciulli, M.; Manna, L.; Brovelli, S. Trap-Mediated Two-Step Sensitization of Manganese Dopants in Perovskite Nanocrystals. ACS Energy Lett. 2019, 4, 85-93.

(54) Das Adhikari, S.; Dutta, A.; Dutta, S. K.; Pradhan, N. Layered Perovskites $\mathrm{L}_{2}\left(\mathrm{~Pb}_{1-\mathrm{x}} \mathrm{Mn}_{\mathrm{x}}\right) \mathrm{Cl}_{4}$ to $\mathrm{Mn}$-Doped $\mathrm{CsPbCl}_{3}$ Perovskite Platelets. ACS Energy Lett. 2018, 3, 1247-1253.

(55) Vink, A. P.; De Bruin, M. A.; Roke, S.; Peijzel, P. S.; Meijerink, A. Luminescence of Exchange Coupled Pairs of Transition Metal Ions. J. Electrochem. Soc. 2001, 148, 313-320.

(56) Ronda, C. R.; Amrein, T. Evidence for Exchange-Induced Luminescence in $\mathrm{Zn}_{2} \mathrm{SiO}_{4}$ : Mn. J. Lumin. 1996, 69, 245248.

(57) Fundamentals of Phosphors; William, M. Y., Shionoya, S., Yamamoto, H., Eds.; CRC Press, 2007.

(58) Biswas, A.; Bakthavatsalam, R.; Kundu, J. Efficient Exciton to Dopant Energy Transfer in $\mathrm{Mn}^{2+}$-Doped $\left(\mathrm{C}_{4} \mathrm{H}_{9} \mathrm{NH}_{3}\right)_{2} \mathrm{PbBr}_{4} \quad$ Two-Dimensional (2D) Layered Perovskites. Chem. Mater. 2017, 29, 7816-7825.

(59) Dutta, S. K.; Dutta, A.; Das Adhikari, S.; Pradhan, N. Doping $\mathrm{Mn}^{2+}$ in Single-Crystalline Layered Perovskite Microcrystals. ACS Energy Lett. 2019, 4, 343-351.

(60) Yeom, T. H.; Lee, Y. H.; Hahn, T. S.; Oh, M. H.; Choh, S. H. Electron-Paramagnetic-Resonance Study of the $\mathrm{Mn}^{2+}$ Luminescence Center in ZnS:Mn Powder and Thin Films. J. Appl. Phys. 1996, 79, 1004-1007.
(61) Van Wieringen, J. S. Paramagnetic Resonance of Divalent Manganese Incorporated in Various Lattices. Discuss. Faraday Soc. 1955, 19, 118-126.

(62) Liu, Y.; Zhang, J.; Han, B.; Wang, X.; Wang, Z.; Xue, C.; Bian, G.; Hu, D.; Zhou, R.; Li, D. S.; Wang, Z.; Ouyang, Z.; Li, M.; Wu, T. New Insights into Mn-Mn Coupling Interaction-Directed Photoluminescence Quenching Mechanism in $\mathrm{Mn}^{2+}$-Doped Semiconductors. J. Am. Chem. Soc. 2020, 142, 6649-6660.

(63) Zelewski, S. J.; Urban, J. M.; Surrente, A.; Maude, D. K.; Kuc, A.; Schade, L.; Johnson, R. D.; Dollmann, M.; Nayak, P. K.; Snaith, H. J.; Radaelli, P.; Kudrawiec, R.; Nicholas, R. J.; Plochocka, P.; Baranowski, M. Revealing the Nature of Photoluminescence Emission in the MetalHalide Double Perovskite $\mathrm{Cs}_{2} \mathrm{AgBiBr}_{6}$. J.Mater. Chem. C 2019, 7, 8350-8356.

(64) Zuo, T.; Sun, Z.; Zhao, Y.; Jiang, X.; Gao, X. The Big Red Shift of Photoluminescence of Mn Dopants in Strained CdS: A Case Study of Mn-Doped MnS-CdS Heteronanostructures. J. Am. Chem. Soc. 2010, 132, 6618-6619.

(65) Yuan, X.; Zheng, J.; Zeng, R.; Jing, P.; Ji, W.; Zhao, J.; Yang, W.; Li, H. Thermal Stability of $\mathrm{Mn}^{2+}$ Ion Luminescence in Mn-Doped Core-Shell Quantum Dots. Nanoscale 2014, 6, 300-307.

(66) Chen, W.; Joly, A. G.; Malm, J. O.; Bovin, J. O.; Wang, S. Full-Color Emission and Temperature Dependence of the Luminescence in Poly-P-Phenylene Ethynylene$\mathrm{ZnS} / \mathrm{Mn}^{2+}$ Composite Particles. J. Phys. Chem. B 2003 107, 6544-6551.

(67) Tanaka, M.; Masumoto, Y. Very Weak Temperature Quenching in Orange Luminescence of $\mathrm{ZnS}: \mathrm{Mn}^{2+}$ Nanocrystals in Polymer. Chem. Phys. Lett. 2000, 324 249-254.

(68) Li, F.; Xia, Z.; Gong, Y.; Gu, L.; Liu, Q. Optical Properties of $\mathrm{Mn}^{2+}$ Doped Cesium Lead Halide Perovskite Nanocrystals: Via a Cation-Anion Co-Substitution Exchange Reaction. J. Mater. Chem. C 2017, 5, 92819287.

(69) Liu, W.; Lin, Q.; Li, H.; Wu, K.; Robel, I.; Pietryga, J. M.; Klimov, V. I. $\mathrm{Mn}^{2+}$-Doped Lead Halide Perovskite Nanocrystals with Dual-Color Emission Controlled by Halide Content. J. Am. Chem. Soc. 2016, 138, 14954 14961.

(70) Chen, D.; Fang, G.; Chen, X. Silica-Coated Mn-Doped $\mathrm{CsPb}(\mathrm{Cl} / \mathrm{Br})_{3}$ Inorganic Perovskite Quantum Dots: Exciton-to-Mn Energy Transfer and Blue-Excitable SolidState Lighting. ACS Appl. Mater. Interfaces 2017, 9 , 40477-40487.

(71) Zhou, G.; Jia, X.; Guo, S.; Molokeev, M.; Zhang, J.; Xia Z. Role of Halogen Atoms on High-Efficiency $\mathrm{Mn}^{2+}$ Emission in Two-Dimensional Hybrid Perovskites. J. Phys. Chem. Lett. 2019, 10, 4706-4712. 


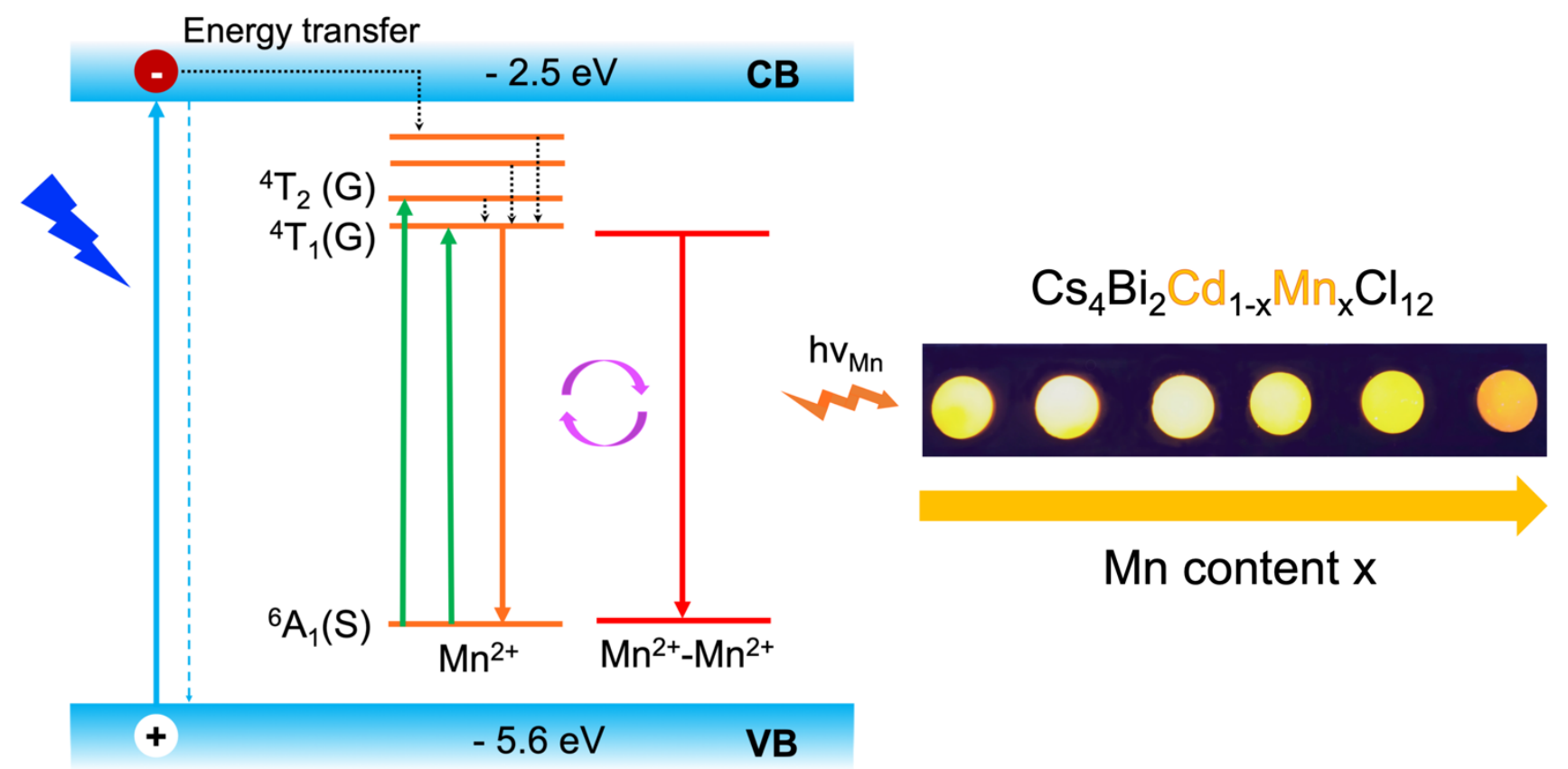

